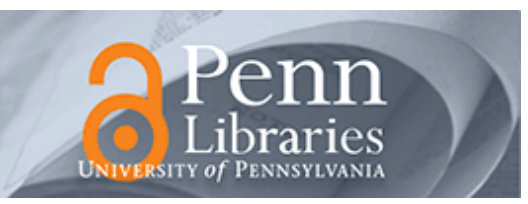

University of Pennsylvania

ScholarlyCommons

July 2005

\title{
Sub-wavelength Focusing and Negative Refraction along Positive- Index and Negative-Index Plasmonic Nano-Transmission Lines and Nano-Layers
}

\author{
Andrea Alù \\ University of Pennsylvania, andreaal@seas.upenn.edu \\ Nader Engheta \\ University of Pennsylvania, engheta@seas.upenn.edu
}

Follow this and additional works at: https://repository.upenn.edu/ese_papers

\begin{abstract}
Recommended Citation
Andrea Alù and Nader Engheta, "Sub-wavelength Focusing and Negative Refraction along Positive-Index and Negative-Index Plasmonic Nano-Transmission Lines and Nano-Layers", . July 2005.
\end{abstract}

Copyright 2005 IEEE. Reprinted from Proceedings of the 2005 IEEE Antennas and Propagation Society International Symposium, Volume 1A, pages 35-38.

This material is posted here with permission of the IEEE. Such permission of the IEEE does not in any way imply IEEE endorsement of any of the University of Pennsylvania's products or services. Internal or personal use of this material is permitted. However, permission to reprint/republish this material for advertising or promotional purposes or for creating new collective works for resale or redistribution must be obtained from the IEEE by writing to pubs-permissions@ieee.org. By choosing to view this document, you agree to all provisions of the copyright laws protecting it.

This paper is posted at ScholarlyCommons. https://repository.upenn.edu/ese_papers/161

For more information, please contact repository@pobox.upenn.edu. 


\title{
Sub-wavelength Focusing and Negative Refraction along Positive-Index and Negative-Index Plasmonic Nano-Transmission Lines and Nano-Layers
}

\author{
Abstract \\ Following our recent works on the concept of plasmonic nano-inductors and nano-capacitors and related \\ complex circuits, here we analyze the possibility of designing nano-transmission-lines (NTL) made of \\ these basic nano-elements. We show that in the limit in which these basic circuit elements are very close \\ to each other, they can be regarded as planar stacks of plasmonic and nonplasmonic slabs, which may be \\ designed to act as forward (right-handed) or backward (lefthanded) NTL. Negative refraction and left- \\ handed propagation are shown to be possible in these planar plasmonic configurations, potentially \\ applicable in several innovative setups for subwavelength focusing, imaging and waveguiding \\ applications.

\section{Comments} \\ Copyright 2005 IEEE. Reprinted from Proceedings of the 2005 IEEE Antennas and Propagation Society \\ International Symposium, Volume 1A, pages 35-38. \\ This material is posted here with permission of the IEEE. Such permission of the IEEE does not in any way \\ imply IEEE endorsement of any of the University of Pennsylvania's products or services. Internal or \\ personal use of this material is permitted. However, permission to reprint/republish this material for \\ advertising or promotional purposes or for creating new collective works for resale or redistribution must \\ be obtained from the IEEE by writing to pubs-permissions@ieee.org. By choosing to view this document, \\ you agree to all provisions of the copyright laws protecting it.
}




\title{
Sub-wavelength Focusing and Negative Refraction along Positive-Index and Negative-Index Plasmonic Nano-Transmission Lines and Nano-Layers
}

\author{
Andrea Alú $^{(1,2)}$, and Nader Engheta ${ }^{(1)}$ \\ (1) University of Pennsylvania, Dept. of Electrical and Systems Engineering, Philadelphia, PA \\ 19104, U.S.A., andrcaalajec.upenn.edu, engheta 9 ect.upenn.edu \\ (2) University of Roma Tre, Dept. of Applied Electronics, Rome, Italy
}

Following our recent works on the concept of plasmonic nano-inductors and nano-capacitors and related complex circuits, here we analyze the possibility of designing nano-transmission-lines (NTL) made of these basic nano-elements. We show that in the limit in which these basic circuit elements are very close to each other, they can be regarded as planar stacks of plasmonic and nonplasmonic slabs, which may be designed to act as forward (right-handed) or backward (lefthanded) NTL. Negative refraction and left-handed propagation are shown to be possible in these planar plasmonic configurations, potentially applicable in several innovative setups for subwavelength focusing, imaging and waveguiding applications.

\section{Introduction}

The anomalous wave interaction with double-negative (DNG) metamaterials (i.e., media in which the real parts of permittivity and permeability are both negative in a given range of frequency) may offer exciting characteristics, with potential applications in design of various devices and components [1-5]. In particular, the negative refraction and resonance effect present at an interface between a DNG material and a common dielectric [1], may be exploited in several interesting ways, e.g., sub-wavelength focusing and 'perfect' lensing [1, 2], or for reducing the dimensions of waveguides and resonators [3]. We have shown in our recent works, for instance, how suitable pairing of DNG materials with common dielectrics may overcome the diffraction limits in different configurations, such as closed and open waveguides [3], transmission through small apertures [4], and scattering and radiation from small objects [5], to name a few. The realization of DNG metamaterials, although achieved by several groups, however, requires technological efforts and challenges at the present time. That is why in some of our works we have proposed, as a valid alternative, to employ $\varepsilon$-negative (ENG) plasmonic materials, whose real part of the permittivity is negative in a certain range of frequency - a property that is exhibited naturally in noble metals at infrared and optical frequencies, and that can be induced relatively easily at lower frequencies with proper inclusions in a host dielectric.

Analyzing the unusual properties of these materials, we have found in particular that open ENG waveguides supporting surface modes may overcome the diffraction limit in their operation [6,7]. Specifically, the more its physical cross section is reduced, the more the fundamental mode remains concentrated around the waveguide itself, in principle providing the possibility of a no-cut-off-thickness mode with vanishing cross section propagating along the waveguide, which may be useful in several applications $[6,7]$.

In a different part of our work, we have recently shown that ENG and dielectric nanoparticles may respectively act as nano-inductors and nano-capacitors in a complex circuit [8]. A natural question following these findings is whether it would be possible to synthesize a nano-transmission-line (NTL) starting from these basic nano-elements. Placing nano-inductors as series elements connected with nano-capacitors in a parallel configuration, in principle one may be able to synthesize such a NTL from its classical circuit representation. However, in the limit in which such elements are closely packed 
together, such a configuration may resemble the geometry we have originally studied to show the anomalies in the surface-wave propagation. Motivated by these analogies, in the following we show how it is indeed possible to synthesize positive-index and negativeindex sub-diffraction NTL by using ENG plasmonic open waveguides, and how such right-handed/left-handed structures may be employed in a similar way as their doublepositive (DPS) and double-negative (DNG) metamaterial counterparts in order to achieve negative refraction and sub-wavelength focusing. This may open doors to interesting potentials in imaging, lensing and waveguiding applications at optical and infrared, as well as microwave frequencies.

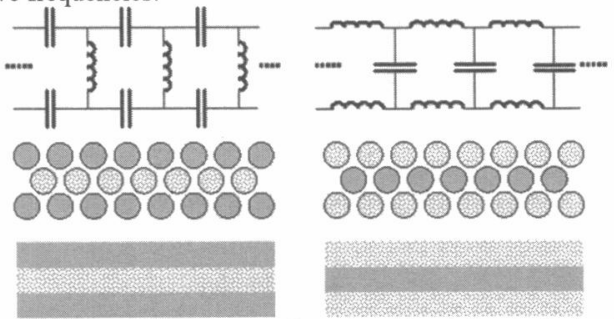

Fig. 1. From [8]. An idea for the implementation of positive-index (right-handed) and negativeindex (left-handed) NTL. (Top row): Conventional RH and LH lines using distributed inductor and capacitor elements; (middle row): Plasmonic and non-plasmonic nanostructures may play the role of nano-inductors and nano-capacitors; (bottom row): As the nanostructures gets closer, in the limit, plasmonic and non-plasmonic layers may be employed.

\section{Nano Transmission-Lines}

An electromagnetic field exciting a dielectric nanosphere induces an electric dipole moment in phase with the impinging electric field. From a circuit point of view this corresponds to having a nano-capacitor driven by a generator [8]. As shown in [8], a plasmonic ENG nanosphere would support a similar argument, but since in this case the induced dipole moment is $180^{\circ}$ out of phase with the excitation, this structure would effectively correspond to a small nano-inductor. These two basic elements, when suitably arranged in a more complex circuit, may be utilized to build positive-index and negativeindex NTL, as depicted in Fig. 1 (top and middle rows). In the figure (top row), the standard circuit models for lossless positive index (left panel) and negative index (right panel) NTL are depicted. In the middle rows the same NTL structure is conceptually synthesized by properly putting together dielectric (darker tones in the figure) and ENG (lighter tones) nanostructures. In the limit in which the nanospheres get closer and closer, the geometries in the middle row of Fig. 1 would tend to form stacks of continuous layers of DPS and ENG materials, which heuristically would show similar properties. This concept is depicted in the bottom row of Fig. 1 for the two cases. The structures may be envisioned as cylindrical rods or planar slabs, giving rise, respectively, to 1-D or 2-D NTL. In the following, we concentrate on the analysis of the planar slabs stacked as in Fig. 1 (bottom row), to show the possibility of right-handed (RH) and left-handed (LH) propagation in such composite 2-D NTL.

\section{Left-Handedness and Negative Refraction in 2-D Nano-Transmission-Lines}

The geometry we refer to is depicted in Fig. 2, together with the corresponding field distribution, for a $\mathrm{RH}$ (on the left) and a $\mathrm{LH}$ (on the right) nano-TL, for the two examples corresponding to the cases of Fig. 1. The materials considered in Fig. 2 are free space and 
isotropic ENG materials, all with permeability $\mu_{0}$, as free space. The thickness of the core slabs is much smaller than the wavelength of operation (around $\lambda_{0} / 100$ in the example of Fig. 2), since, as discussed in [7], this geometry supports no cut-off modes (with propagating factor $e^{-j \beta x}$ ), with highly confined guided modes around the interfaces between dielectrics and ENG materials when the slabs are sufficiently thin. In particular, for the case at hand, we are interested in the even mode of operation, which follows the heuristic analogy of Fig. 1. We note how the field distributions "resemble" those of a standard TL, with "currents" concentrated in the region near the two interfaces. In this limit of sub-wavelength NTL, it can be shown that the dispersion relation for these even modes may be written in the simplified form $\beta d=-\tanh ^{-1} \varepsilon_{\text {core }} / \varepsilon_{\text {shell }}$, where $d$ is the thickness of the core slab, and this justifies why isotropic ENG materials are needed to support such anomalous sub-diffraction modes, and why these modes are slow-wave ( $\beta \gg k_{0}$, with $k_{0}$ being the free-space wavenumber, see the caption of Fig. 2).

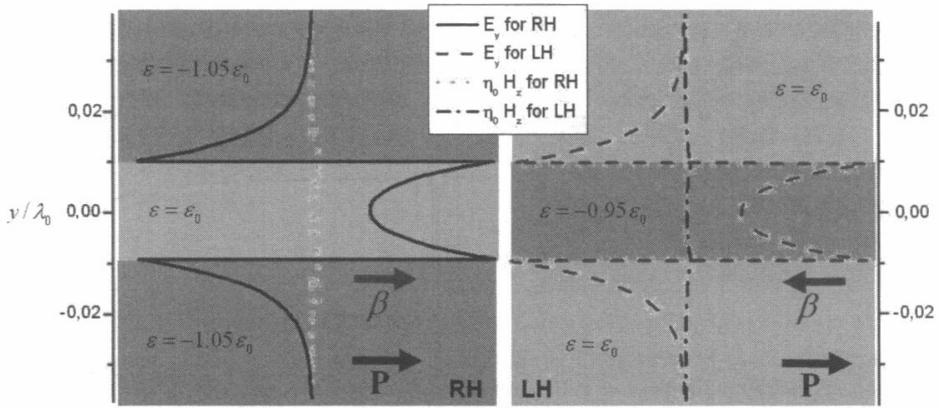

Fig. 2. Field distribution for the dominant even mode in the two cases of RH and LH NTL (cross section is shown here). The parameters have been chosen to have impedance-matched nano-layers, and in the two cases $\beta_{R H}=29.5 k_{0}, \beta_{L H}=-28.9 k_{0}$. The $\mathbf{P}$ vector indicates the direction of power propagation, whereas the $\beta$ vector refers to the phase flow.

The values of the negative epsilon and the thickness of the two ENG layers for the RH and LH nano-layers here have been chosen such that we have similar field distributions in both segments, even though the phase velocities in the two nano-TL are anti-parallel with each other. (We have performed a detailed analysis for this design, but due to the lack of space it is not shown here.) This choice implies a very low reflection at the interface between such two planar waveguides, since the two modes have very similar characteristic impedance in this case. In Fig. 3 (left panel) we have reported the top view of the phase distribution in the case of an interface between two such NTL, in which one impinging mode, coming from the left in the RH nano-layer and carrying power obliquely with respect to the interface experiences negative refraction and (almost) total transmission into the LH nano-layer on the right (the reflection coefficient at the interface is around $5 \%$ ). We reiterate that this phenomenon, analogous to what has been obtained at microwave frequencies with planar 2-D TL loaded with lumped circuit elements [9, 10], has been obtained here, by using plasmonic isotropic ENG metamaterial layers, without any need for negative-permeability materials, and potentially at frequencies (e.g., IR and visible regimes) where standard lumped circuit elements may not be easily feasible. The potential applications of this structure may span from sub-wavelength focusing and near-field imaging to diffraction-less transport of information. (We point out that there are other approaches to achieve negative refraction without using magnetic 
materials, see e.g. [11-15]). In Fig. 3 (right panel) the transmission coefficient of the interface as a function of $\beta_{z} / k_{0}$ is shown. Here the values of $\beta_{z}$ (transverse component of $\beta$ with respect to the interface) for which a very high transmission is possible at this interface is sensibly higher than $k_{0}$; however, we need to remember that the value of wavenumber of the guided mode is already much higher than $k_{0}$ (e.g., for our design, $\beta_{R H}=29.5 k_{0}, \beta_{L H}=-28.9 k_{0}$ ). Nevertheless, the plot of transmission coefficient shows the realistic possibility of sub-wavelength focusing with this setup, since when $\beta_{z}$ becomes greater than $\beta_{L H}$, the "growing exponential" phenomenon $[1,2]$ may be achieved, as evident form the growth of the transmission coefficient in this evanescent region. More details and physical insights into this phenomenon will be mentioned during the talk.
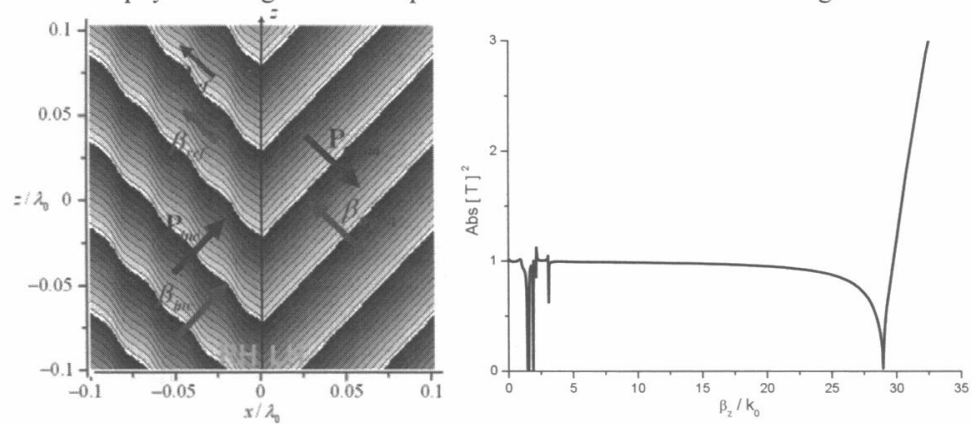

Fig. 3. (Left): Interface between the RH and LH nano-TL of Fig. 2 (contour plot of the phase distribution, top view). Note the low reflection at the interface, the nearly complete transmission, and the clearly evident negative refraction at the interface, which underlines the left-handedness of the second NTL; (right): Transmission coefficient at the interface as a function of the transverse $\beta_{z}$ (i.e., the angle of incidence). Note the growing transmission coefficient in the evanescent region, connected to the growing-exponential and sub-wavelength focusing phenomenon $[1,2]$.

\section{References}

[1] A. Alù, and N. Engheta, IEEE Trans. Antennas Propagat., 51, 2558 (2003).

[2] J. B. Pendry, Phys. Rev. Lett., 85, 3966 (2000).

[3] A. Alù, and N. Engheta, IEEE Trans. Microwave Theory \& Tech., 52, 199 (2004).

[4] A. Alù, F. Bilotti, N. Engheta, and L. Vegni, submitted to IEEE Trans. Antennas Propagat., under review. (The manuscript can be viwed at http://arxiv.org/pdf/cond-mat/0408582.)

[5] A. Alù, and N. Engheta, submitted for publication, under review. (The manuscript can be viewed at http://arxiv.org/pdf/physics/0410011.)

[6] A. Alù, and N. Engheta, Proc. of PIERS'04, Pisa, Italy, CD Digest, March 28-31, 2004

[7] A. Alù, and N. Engheta, in G. V. Eleftheriades, and K. G. Balmain, Negative Refraction Metamaterials: Fundamental Properties and Applications, John Wiley \& Sons, Inc., 2005.

[8] N. Engheta, A. Salandrino, and A. Alù, submitted for publication, under review. (The manuscript can be viewed at: http://arxiv.org/pdf/cond-mat/0411463.)

[9] G. V. Eleftheriades, A. K. Iyer, and P. C. Kremer, IEEE Trans. MTT, 50, 2702 (2002).

[10] L. Liu, C. Caloz, C.-C. Chang, and T. Itoh, J. Appl. Phys., 92, 5560 (2002).

[11] S. O'Brien, et al., Phys. Rev. B, 69, 241101 (2004).

[12] G. Shvets, and Y. A. Urzhumov, Phys. Rev. Lett., 93, 243902 (2004).

[13] G. Shvets, Phys. Rev. B. 67, 035109 (2003).

[14] M. L. Povinelli, et al., Appl. Phys. Lett., 82, 1069 (2003).

[15] V. A. Podolskiy, and E. E. Narimanov, online at http://arxiv.org/pdf/physies/0405077. 